\title{
Thermalization time and specific heat of neutron stars crust
}

\section{J. Margueron ${ }^{* \dagger}$}

Institut de Physique Nucl'eaire, IN2P3-CRNS and Universit'e Paris-Sud, France

E-mail: jerome.margueron@ipno.in2p3.fr

We discuss the thermalization process of the neutron stars crust described by solving the heat transport equation with a microscopic input for the specific heat of baryonic matter. The heat equation is solved with initial conditions specific to a rapid cooling of the core. To calculate the specific heat of inner crust baryonic matter, i.e., nuclear clusters and unbound neutrons, we use the quasiparticle spectrum provided by the Hartree-Fock-Bogoliubov approach at finite temperature. In this framework we analyze the dependence of the crust thermalization on pairing properties and on cluster structure of inner crust matter. It is shown that the pairing correlations reduce the crust thermalization time by a large fraction. The calculations show also that the nuclear clusters have a non-negligible influence on the time evolution of the surface temperature of the neutron star.

XXXIV edition of the Brazilian Workshop on Nuclear Physics

5-10 June 2011

Foz de Iguanu, Parana state, Brasil

* Speaker.

$\dagger$ A footnote may follow. 
The thermalization process of the neutron stars crust can give important informations concerning the properties of the crust matter. This is indeed the case in the cooling of isolated neutron stars [1] and in the thermal after-burst relaxation of neutron stars from X-ray transients [2,3]. In the cooling process an important quantity is the cooling or thermalization time of the crust, defined as the time needed for the crust matter to equilibrate its temperature to the temperature of the colder core. The outer crust, due to its thinness and the very small amount of matter it contains, has a very short thermal time-scale. Hence, the cooling time is essentially determined by the inner crust thickness, as well as by the composition of the inner crust matter formed by nuclear clusters, unbound neutrons and ultra-relativistic electrons [1]. Moreover several studies have shown that the thermalization time depends significantly on the superfluid properties of the inner crust baryonic matter $[1,4,5,6,7]$. This dependence is induced through the specific heat of unbound neutrons, strongly affected by the pairing energy gap. Since the neutron pairing gap is influenced by the presence of the nuclear clusters $[8,5,9]$, a reliable calculation of neutron specific heat should take the clusters into account. How the intensity of pairing correlations affects the specific heat of the neutrons in the presence of nuclear clusters was analyzed in Ref. [10]. Using the framework of the Hartree-Fock-Bogoliubov (HFB) approach at finite temperature it was shown that the specific heat can change by several orders of magnitude if the pairing gap is adjusted to describe two possible scenarios for neutron matter superfluidity, i.e., one corresponding to BCS approximation and the other to calculations schemes which take into account in-medium effects [11]. The impact which these changes in the specific heat could have on the thermalization time was discussed in Ref. [6]. Employing a simple random walk model for the cooling [5, 12], in which the diffusion of the heat towards the core was calculated without taking into account the dynamical change of the temperature through the whole crust, it was shown that the thermalization times corresponding to the two pairing scenarios mentioned above differ by a large fraction. The scope of this work is to perform more accurate estimations of the thermalization time obtained by employing a more realistic cooling model based on dynamical solutions of the heat equations [13] and on a state-of-the-art description of the specific heat for baryonic matter.

\section{Specific heat of the inner crust matter}

The specific heat of the inner crust has contributions from electrons, the lattice and unbound neutrons. They are calculated for a given set of densities in the Wigner-Seitz approach. In the inner crust the unbound neutrons are superfluid in the ${ }^{1} \mathrm{~S}_{0}$ channel. To take into account the superfluid properties of the neutrons as well as the non-uniform cluster structure of the inner crust, we perform HFB calculations at finite-temperature for each Wigner-Seitz cell [15]. The details of the HFB calculations in a Wigner-Seitz cell are given in Ref. [10]. In the HFB calculations the mean field is described with the same interaction used in the Douchin and Haensel EoS [16], i.e., the Skyrme force SLy4.

We present now in more detail the results for the cell which contains $\mathrm{N}=460$ neutrons and $\mathrm{Z}=40$ protons [15]. In this cell the HFB calculations predict 378 unbound neutrons. It is interesting that in spite of the large number of neutrons in the cell, the number of the bound neutrons in the cluster with $\mathrm{Z}=40$ protons is equal to the magic number 82 , as for the dripline nucleus ${ }^{122} \mathrm{Zr}$ (see, e.g., Ref. [17]). The specific heat given by the HFB spectrum, in which the contribution of the 


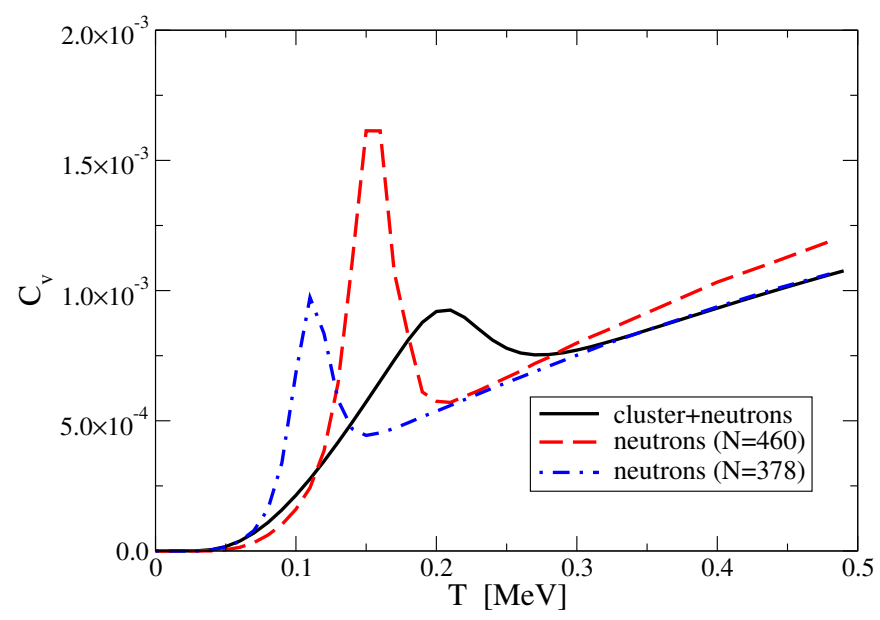

Figure 1: Neutron-specific heat in the the Wigner-Seitz cell $N=460$ neutrons and $Z=40$ protons [15] for strong pairing. The results corresponds to various approximations discussed in the paper. The specific heat is given in units of Boltzman constant $k_{B}$.

cluster is included, is shown in Fig. 1 by the full line. In the same figure are shown the specific heat corresponding to two approximations employed in some studies [1,5]: the uniform distribution of neutrons is calculated in the WS cell removing all the protons. The number of neutrons is fixed to be equal either to the total number of neutrons in the cell (dashed line) or only to the number of the unbound neutrons (dash-dotted line). The latter case is considered as an effective way of taking into account the influence of the cluster [5]. How these approximations work is seen in Fig. 1. To make the comparison meaningful, the calculations for the uniform neutron gas are done solving the HFB equations with the same boundary conditions as for the non uniform system, i.e., neutrons+cluster. As seen in Fig. 1, the transition from the superfluid to the normal phase, localized at the peak of the curves, takes place at a lower temperature in the case of uniform neutron gas, especially when are considered only the unbound neutrons. We can also notice that, in contrast to the uniform system, in the non uniform system the transition from the superfluid to the normal phase is smooth.

To see better what happens in a non uniform system, in Fig. 2 (left) is shown the evolution with the temperature of the pairing field in the same cell as in Fig. 1. The pairing field is defined by

$$
\Delta(r)=V_{0}\left[1-\eta\left(\frac{\rho(r)}{\rho_{0}}\right)^{\alpha}\right] \kappa(r)
$$

where $\kappa(r)$ is the local pairing tensor. Notice that the pairing field is taken with negative values. From Fig. 2 (left) we can see that at zero temperature the pairing field is much larger at the surface of the cluster than in the bulk region. Due to this fact by increasing the temperature the pairs corresponding to the neutrons localized preferentially at the surface region of the cluster are destroyed gradually and much slower compared with the pairs formed by neutrons localized far from the surface of the cluster. In fact, as shown in Fig. 2 (right), the non uniform system shows two transition regions, one around $\mathrm{T}=200 \mathrm{keV}$, corresponding mainly to the neutrons located far from the surface of the cluster, and another one, much less pronounced, around $\mathrm{T}=900 \mathrm{keV}$, which corresponds to the neutrons localized in the surface region of the cluster. 

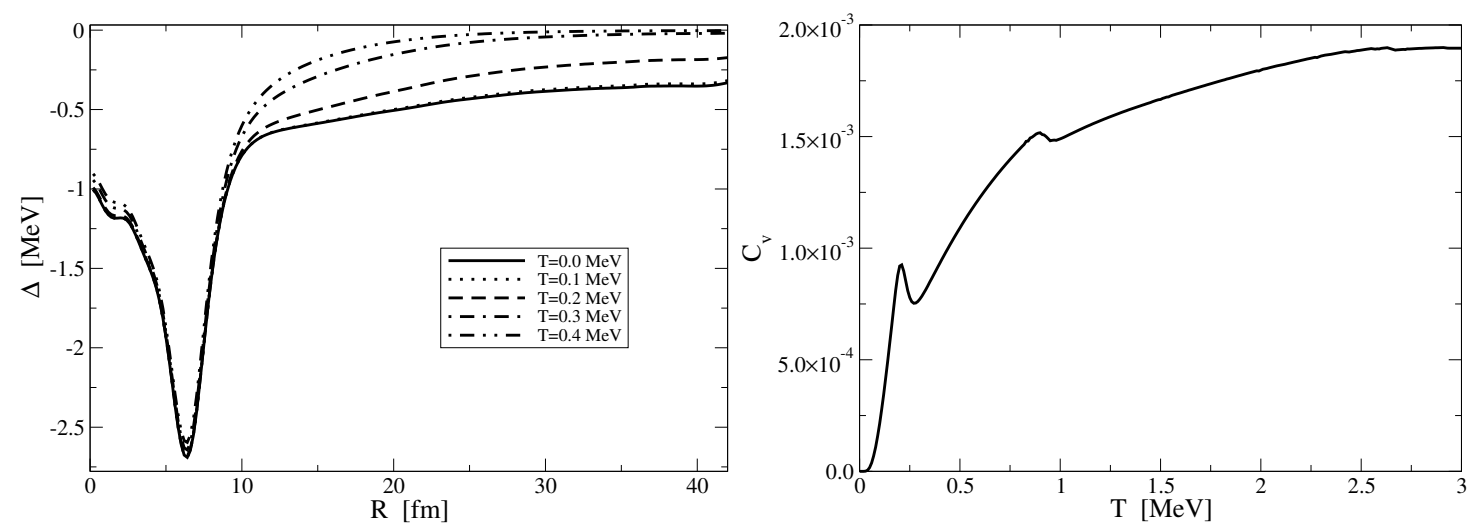

Figure 2: On the left is shown the radial distribution of the pairing field for the Wigner-Seitz cell $N=460$ neutrons and $\mathrm{Z}=40$ protons and for various temperatures. The results correspond to a strong pairing force. On the right is represented the neutron-specific heat for the same Wigner-Seitz cell. The results are shown up to high temperatures in order to illustrate the second transition region around $\mathrm{T}=900 \mathrm{keV}$. The specific heat is given in units of $k_{B}$.
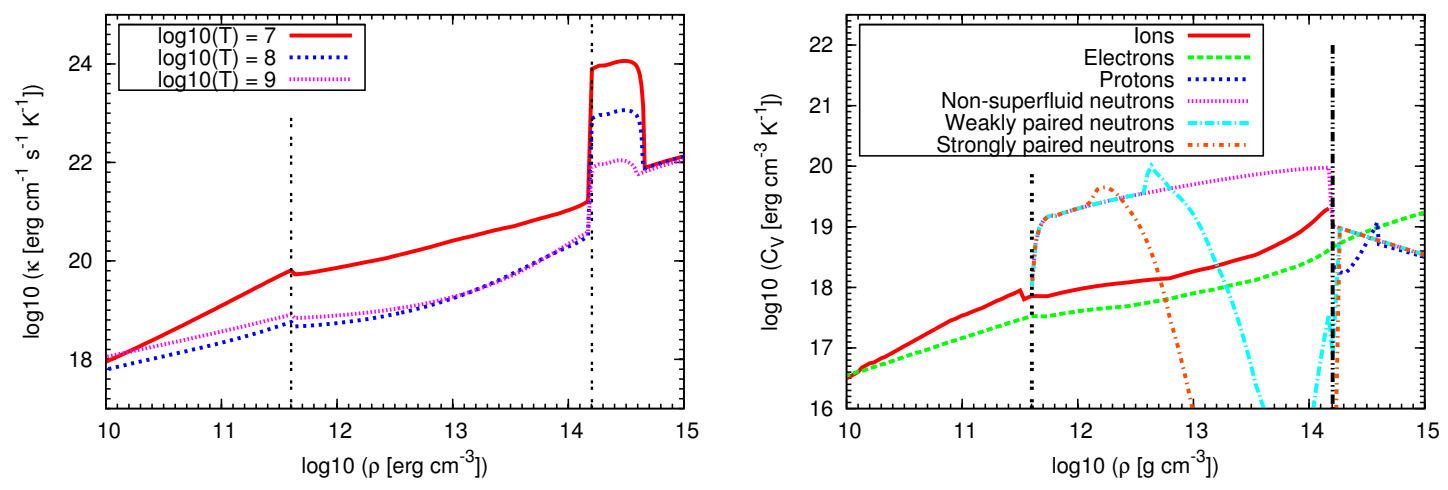

Figure 3: On the left the total conductivity in the neutron star is shown as a function of the density and for the temperatures $T=\left\{10^{7}, 10^{8}, 10^{9}\right\} \mathrm{K}$ (see the text for more details). The bounds of the inner crust are indicated by the vertical lines. On the right the specific heat in the crust of the neutron star is represented for the different components of the star matter and for a temperature of $10^{9} \mathrm{~K}$ (see the text for more details).

\section{Crust thermalization}

The cooling process is described by solving the heat equation in the whole volume of the neutron star (more details and references can be found in Ref. [14]). The initial temperature distribution in the star is chosen to be constant, $T(r, t=0)=T_{i}$. The cooling calculations presented here are for a neutron star of a mass equal to $1.6 M_{\odot}$. For this mass and for the Douchin and Haensel EoS [16] the TOV equations predict a total star radius of $11.49 \mathrm{~km}$ and a central density of $4.06 \rho_{0}$, where $\rho_{0}=2.9 \times 10^{14} \mathrm{~g} \mathrm{~cm}^{-3}$ is the saturation density of symmetric nuclear matter. The three needed microphysical ingredients, the conductivity $K$, the specific heat $C_{V}$, and the neutrino emission rate $Q_{v}$, as well as pairing of neutron and protons in the core, are described in Ref. [14]. 

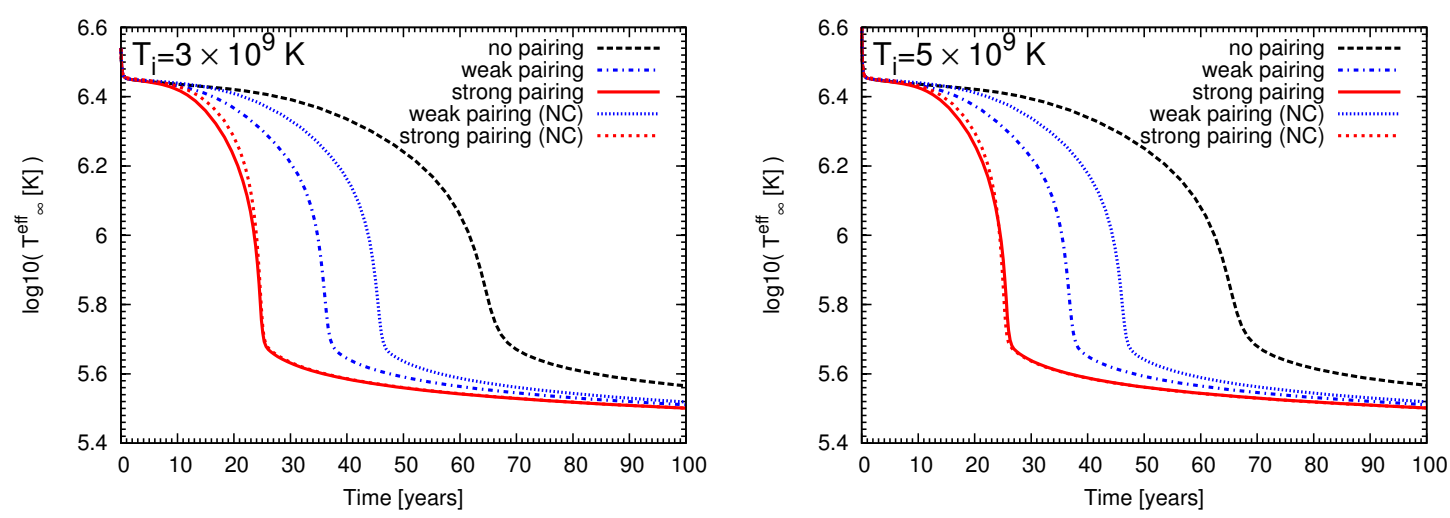

Figure 4: Time evolution of the apparent surface temperature for the initial temperatures $T_{i}=$ $\{300,500\} \mathrm{keV}$. NC indicates the results of the calculations obtained by neglecting the effect of the clusters.

The overall dependence of $K$ on density is shown in Fig. 3 (left) for three temperatures, i.e., $T=\left\{10^{7}, 10^{8}, 10^{9}\right\} \mathrm{K}$, typical for the cooling calculations performed in this study. In the crust the conductivity shown in Fig. 3 (left) is similar to that of Ref. [4]. In the core Fig. 3 (left) clearly exhibits the $\propto T^{-1}$ behavior at densities $\rho<5 \times 10^{14} \mathrm{~g} \mathrm{~cm}^{-3}$ where protons are superconductors, contrasted by the T-independent values at higher densities. This high density behavior of $K^{\mathrm{e}}$ was not included in the work of Ref. [4] which was previous to Ref. [18].

The dependence of the specific heat on the density is illustrated in Fig. 3 (right) for a temperature equal to $10^{9} \mathrm{~K}$. In the outer crust the specific heat has contributions from electrons and ions while in the core from electrons, neutrons and protons. These specific heats are evaluated using standard approximations (see, e.g., [19]). The specific heat is strongly dependent on the physical state of the neutrons in the inner crust. Indeed, the pairing strongly supress the contribution of the neutrons to the specific heat which otherwise would give a dominant contribution, see Fig. 3 (right). In the region where neutrons are paired the ions provide the major contribution to the specific heat, eventually being supplanted by the electrons since $C_{V}^{(\text {ion })} \propto T^{3}$ while $C_{V}^{(\mathrm{e})} \propto T$.

The time evolution of the effective surface temperature $T_{\infty}^{\text {eff }}$ is displayed in Fig. 4 for two initial temperatures of the crust, $T_{i}=\{300,500\} \mathrm{keV}$. The effective surface temperature shown in Fig. 4 is obtained from the temperature at the bottom of the crust, $T_{\mathrm{b}}=T\left(\rho_{\mathrm{b}}\right)$, where $\rho_{\mathrm{b}}=10^{10}$ $\mathrm{g} \mathrm{cm}^{-3}$, using the relationship given in Ref. [20] for a non-accreted envelope. It can be seen that the time evolution of the surface temperature does not depend much on the initial temperature of the crust. In Fig. 4 the apparent surface temperatures obtained by neglecting the effect of the clusters is shown, i.e., supposing that the neutron specific heat is given solely by that of the neutron gas. In this case the specific heat of the neutrons is calculated from the quasiparticle spectrum of BCS equations solved for infinite neutron matter at a density corresponding to that of the external neutrons in the WS cell [21]. It can be seen that for the weak pairing scenario the clusters have a non-negligible effect on the time evolution of the surface temperature. For the strong pairing the initial temperature being already lower than the critical temperature there is almost no impact of the cluster on the thermal relaxation. 


\section{Conclusion}

In this work we have studied how the thermalization of neutron stars crust depends on pairing properties and the cluster structure of the inner crust matter. The thermal evolution was obtained by solving the relativistic heat equation with initial conditions specific to a rapid cooling process. The specific heat of neutrons was calculated from the HFB spectrum, taking into account consistently the effects of nuclear clusters, pairing correlations and temperature. The thermal evolution of the inner crust was analyzed using for the neutrons two sets of specific heats obtained with a strong and a weak pairing force which simulate two possible scenarios for the intensity of pairing correlations in neutron matter. The results show that the crust thermalization is strongly influenced by the intensity of pairing correlation. We have also shown that the cluster structure of the inner crust affects significantly the time evolution of the surface temperature.

Acknowledgment. This work was supported by ESF through the project " The New Physics of Compact Stars", by the ANR NExEN, and by CNCSIS through the grant IDEI no. 270.

\section{References}

[1] J. M. Lattimer, K. A. Van Riper, M. Prakash, and M. Prakash, Astrophys. J. 425, 802 (1994)

[2] P. S. Shternin, D. G. Yakovlev, P. Haensel, and A. Y. Potekhin, Mont. Not. R. Astron. Soc. 382, L43 (2007)

[3] E. F. Brown, and A. Cumming, Astrophys. J. 698, 1020 (2009)

[4] O. Y. Gnedin, D. G. Yakovlev, A. Y. Potekhin, Mont. Not. R. Astron. Soc. 324, 725 (2001)

[5] P.M. Pizzochero, F. Barranco, E. Vigezzi, and R. A. Broglia, Astrophys. J. 569, 381 (2002)

[6] C. Monrozeau, J. Margueron, and N. Sandulescu, Phys. Rev. C 75, 065807 (2007)

[7] N. Sandulescu, Eur. Phys. J. (Special Topics) 156, 265 (2008)

[8] F. Barranco, R. A. Broglia, H. Esbensen, and E. Vigezzi, Phys. Rev. C 58, 1257 (1998)

[9] N. Sandulescu, N. Van Giai, and R. J. Liotta, Phys. Rev. C 69, 045802 (2004)

[10] N. Sandulescu, Phys. Rev. C 70, 025801 (2004)

[11] U. Lombardo, in Nuclear Methods and the Nuclear Equation of State, edited by M. Baldo (World Scientific, Singapore, 1999), pp. 458-510

[12] G. E. Brown, K. Kubodera, D. Page, and P. M. Pizzochero, Phys. Rev. D 37, 2042 (1988)

[13] D. Page, U. Geppert \& F. Weber, Nucl. Phys. A 777, p. 497-530 (2006); D. Page \& S. Reddy, Annu. Rev. Nucl. \& Part. Sci. 56, 327 (2006)

[14] M. Fortin, F. Grill, J. Margueron, D. Page and N. Sandulescu, Phys. Rev. C 82, 065804 (2010)

[15] J. W. Negele and D. Vautherin, Nucl. Phys. A 207, 298 (1973)

[16] F. Douchin and P. Haensel, Astron. Astrophys. 380, 151167 (2001)

[17] N. Sandulescu, L. S. Geng, H. Toki, and G. C. Hillhouse, Phys. Rev. C 68, 054323 (2003)

[18] P. S. Shternin, and D. G. Yakovlev, Phys. Rev. D 75, 103004 (2007) 
[19] D. Page, J. M. Lattimer, M. Prakash, and A.W. Steiner, Astrophys. J. Suppl. 155, 623 (2004)

[20] A. Y. Potekhin, G. Chabrier, and D. G. Yakovlev, Astron. Astrophys. 323, 415 (1997)

[21] K. P. Levenfish, D. G. Yakovlev, Astron. Rep. 38, 247 (1994) 